\title{
Discrete spectrum of perturbed Dirac systems with real and periodic coefficients
}

\section{Boris Buffoni}

Département de Mathématiques, Ecole Polytechnique Fédérale de Lausanne, 1015 Lausanne, Switzerland(MS received 16 November 1989. Revised MS received 12 February 1990)

\section{Synopsis}

This paper deals with the number of eigenvalues which appear in the gaps of the spectrum of a Dirac system with real and periodic coefficients when the coefficients are perturbed. The main results provide an upper bound and a condition under which exactly one eigenvalue appears in a given gap.

\section{Introduction}

Let us consider the differential expression

$$
\sigma y(x)=-y^{\prime \prime}(x)+q(x) y(x),
$$

where $y$ is a complex valued function defined on $\mathbb{R}$ and $q: \mathbb{R} \rightarrow \mathbb{R}$ is periodic with period $a>0$ and locally absolutely integrable.

The maximal operator $S$ generated by $\sigma$ on $\mathbb{R}[7]$ is self-adjoint and is called the Hill's operator. Its spectrum $\sigma(S) \subset \mathbb{R}$ is purely continuous, bounded from below but unbounded from above, and it is a locally finite union of closed intervals of positive length. In the following, we shall suppose that $\sigma(S)$ has an infinity of gaps; this is so, for example, if $q \in L_{\text {loc }}^{2}(\mathbb{R})$ and is not analytic [5].

Rofe-Beketov [3,4] studied the perturbed Hill's operator $\tilde{S}$, which is the maximal operator generated on $\mathbb{R}$ by the differential expression

$$
\tilde{\sigma} y(x)=-y^{\prime \prime}(x)+\{q(x)+\Delta q(x)\} y(x),
$$

where $\Delta q: \mathbb{R} \rightarrow \mathbb{R}$ is such that $|\Delta q(x)|(1+|x|)$ is integrable on $\mathbb{R}$. This is a self-adjoint operator with the same essential spectrum $\sigma_{e}(\tilde{S})$ as $S$. He proved that there is only a finite number of eigenvalues of $\tilde{S}$ in each gap an at most two eigenvalues in each sufficiently remote gap; moreover, there is exactly one eigenvalue in each sufficiently remote gap if the following additional condition is satisfied: $\int \Delta q(x) d x \neq 0$.

The purpose of this paper is to prove analogous results for Dirac systems. Let $\tau$ be the differential expression

$$
\tau u(x)=R(x)^{-1}\left\{\left(\begin{array}{rr}
0 & 1 \\
-1 & 0
\end{array}\right) u^{\prime}(x)+P(x) u(x)\right\},
$$

where $u$ is a $\mathbb{C}^{2}$-valued function defined on $\mathbb{R} ; P$ and $R$ are symmetric $2 \times 2$ matrices, with locally absolutely integrable real entries which are periodic with period $a>0 ; R$ is positive definite almost everywhere; and let $L^{2}(] c, d[, R)$ be 
the Hilbert space defined by

$$
L^{2}(] c, d[, R)=\{u:] c, d\left[\rightarrow \mathbb{C}^{2} \mid \int_{c}^{d}(R(x) u(x), u(x)) d x<\infty\right\}
$$

and

$$
\langle u, v\rangle=\int_{c}^{d}(R(x) u(x), v(x)) d x,
$$

where $(.,$.$) denotes the usual scalar product in \mathbb{C}^{2}$ and $-\infty \leqq c<d \leqq+\infty$ (we do not distinguish between two functions which are equal almost everywhere). The maximal operator $T$ generated by $\tau$ and defined in $L^{2}(\mathbb{R}, R)$ is self-adjoint, its spectrum $\sigma(T)$ is purely continuous, unbounded from above and below, and it is a locally finite union of closed intervals of positive length [7, theorem 12.5]. In the following, we shall suppose that $\sigma(T)$ has at least one gap; for example, if

$$
a=2, \quad R \equiv\left(\begin{array}{ll}
1 & 0 \\
0 & 1
\end{array}\right),\left.\quad P\right|_{\jmath 0,1 \mid} \equiv\left(\begin{array}{rr}
-2 & 0 \\
0 & 0
\end{array}\right),\left.\quad P\right|_{] 1,2[} \equiv\left(\begin{array}{ll}
0 & 0 \\
0 & 2
\end{array}\right),
$$

there is an infinity of gaps [7, chap. 17.G].

We shall examine the perturbed operator $\tilde{T}$, which is the maximal operator generated on $\mathbb{R}$ by the differential expression

$$
\tilde{\tau} u(x)=R(x)^{-1}\left\{\left(\begin{array}{rr}
0 & 1 \\
-1 & 0
\end{array}\right) u^{\prime}(x)+\{P(x)+\Delta P(x)\} u(x)\right\},
$$

where $\Delta P(x)$ is a symmetric $2 \times 2$ matrix, with absolutely integrable real entries, and whose support satisfies $\operatorname{supp}(\Delta P) \subset[A, B](-\infty<A \leqq B<+\infty)$. The operator $\tilde{T}$ is self-adjoint and $\sigma_{e}(\tilde{T})=\sigma_{e}(T)$; this can be proved by the method of decomposition [1].

Let $] \mu, v\left[\right.$ be a gap of $\sigma_{e}(\tilde{T})$, let $r_{2}(x), p_{2}(x), \delta p_{2}(x)$ be the largest eigenvalues of respectively $R(x), P(x), \Delta P(x)$, and let $r_{1}(x), p_{1}(x), \delta p_{1}(x)$ be the corresponding lowest eigenvalues. We shall show that if for $N \in \mathbb{N}$,

$$
\int_{A}^{B}\left\{(|\mu|+|v|)\left(r_{2}(x)-r_{1}(x)\right)+2\left(p_{2}(x)-p_{1}(x)\right)+\left(\delta p_{2}(x)-\delta p_{1}(x)\right)\right\} d x \leqq N \pi,
$$

then there are at most $N+1$ eigenvalues of $\tilde{T}$ in $] \mu, v[$, and if

$$
\begin{gathered}
\int_{A}^{B}\left\{\max (|\mu|,|v|)\left(r_{2}(x)-r_{1}(x)\right)+\left(p_{2}(x)-p_{1}(x)\right)+\left|\delta p_{2}(x)\right|\right\} d x \leqq \pi / 2, \\
\delta p_{1}(x)=\delta p_{2}(x) \quad \text { almost everywhere on } \mathbb{R},
\end{gathered}
$$

$\delta p_{2}$ is not equal almost everywhere to the null function,

$$
\delta p_{2}(x) \geqq 0 \quad \text { almost everywhere or } \delta p_{2}(x) \leqq 0 \text { almost everywhere on } \mathbb{R} \text {, }
$$

then there is exactly one eigenvalue in $] \mu, v[$.

But first we shall present an important tool, which is an adaptation of the oscillation theory for Dirac systems developed by Weidmann [7, chap. 16]. 


\section{Oscillation theory for Dirac systems defined on $\mathbb{R}$ and which are in the limit point case at $-\infty$ and $+\infty$}

Let $\tau$ be the differential expression

$$
\tau u(x)=R(x)^{-1}\left\{2\left(\begin{array}{cc}
0 & q(x) \\
-q(x) & 0
\end{array}\right) u^{\prime}(x)+\left(\begin{array}{cc}
0 & q^{\prime}(x) \\
-q^{\prime}(x) & 0
\end{array}\right) u(x)+P(x) u(x)\right\},
$$

where $u$ is a $\mathbb{C}^{2}$-valued function defined on $\mathbb{R} ; P$ and $R$ are symmetric $2 \times 2$ matrices, with locally absolutely integrable real entries; $R$ is positive definite almost everywhere; $q$ is a locally absolutely continuous real valued function and for all values of $x \in \mathbb{R}: q(x)>0$. If $u$ is a non-trivial real solution of $\tau u=\lambda u$, we introduce the transformation

$$
u(x)=\rho(x)\left(\begin{array}{l}
\cos \theta(x) \\
\sin \theta(x)
\end{array}\right), \quad \rho(x)>0,
$$

where $\rho$ and $\theta$ are continuous and $\theta$ is defined up to $2 k \pi$. If $\theta$ is completely defined (for instance, if we know its value at a given $x_{0}$ ), we shall call it $a$ determination of the angular part of $u$.

It is easy to check that

with

$$
\theta^{\prime}(x)=\left(G(x)\left(\begin{array}{c}
\cos \theta(x) \\
\sin \theta(x)
\end{array}\right),\left(\begin{array}{c}
\cos \theta(x) \\
\sin \theta(x)
\end{array}\right)\right)
$$

$$
G(x)=\frac{1}{2 q(x)}(\lambda R(x)-P(x)) .
$$

We write $\theta(\lambda, \alpha, c, x)(\lambda, \alpha, c$ in $\mathbb{R})$ for the angular part which

(i) corresponds to the solution $v(x)=\left(v_{1}(x), v_{2}(x)\right)$ of $\tau u=\lambda u$, satisfying $v_{1}(c)=\cos (\alpha)$ and $v_{2}(c)=\sin (\alpha)$;

(ii) is such that $\theta(\lambda, \alpha, c, c)=\alpha$.

Proposition 1.1. If the maximal operator $T$ generated by $\tau$ on $\mathbb{R}$ is self-adjoint and $-\infty<\mu<\lambda<+\infty$, then

$$
\operatorname{dim} R\left(E_{T}(\lambda-)-E_{T}(\mu)\right) \leqq \liminf _{n \rightarrow \infty}\left[\frac{\theta\left(\lambda, \alpha_{n}, c_{n}, d_{n}\right)-\theta\left(\mu, \alpha_{n}, c_{n}, d_{n}\right)}{\pi}\right],
$$

where $\left(\alpha_{n}\right) \subset \mathbb{R}, \quad c_{n} \rightarrow-\infty, d_{n} \rightarrow+\infty, E_{T}($.$) is the right continuous spectral$ resolution of $T$ and $[x]=\max \{k \in \mathbb{Z} \mid k \leqq x\}$.

Proof. Let $\delta>0$ be such that $\mu+\delta$ and $\lambda-\delta$ are not eigenvalues of $T$ and $\mu+\delta<\lambda-\delta$, let $\beta_{n}$ be defined by $\beta_{n}=\theta\left(\mu+\delta, \alpha_{n}, c_{n}, d_{n}\right)$ and let us define the self-adjoint operators $B_{n}, O_{n}$ and $T_{n}$ as follows:

with

$$
B_{n}:\left\{\begin{array}{l}
D\left(B_{n}\right) \subset L^{2}(] c_{n}, d_{n}[, R) \rightarrow L^{2}(] c_{n}, d_{n}[, R), \\
B_{n} u=\tau u,
\end{array}\right.
$$

$$
\begin{gathered}
D\left(B_{n}\right)=\left\{u \in L^{2}(] c_{n}, d_{n}[, R) \mid u \text { is loc. abs. cont., } \tau u \in L^{2}(] c_{n}, d_{n}[, R)\right. \text { and } \\
\left.\sin \left(\alpha_{n}\right) u_{1}\left(c_{n}\right)-\cos \left(\alpha_{n}\right) u_{2}\left(c_{n}\right)=\sin \left(\beta_{n}\right) u_{1}\left(d_{n}\right)-\cos \left(\beta_{n}\right) u_{2}\left(d_{n}\right)=0\right\}
\end{gathered}
$$


The sequence $\left(T_{n}\right)$ converges to $T$ in the sense of the strong resolvent convergence and therefore

$$
\begin{aligned}
\operatorname{dim} R\left(E_{T}(\lambda-\delta)-E_{T}(\mu+\delta)\right) & \leqq \liminf _{n \rightarrow \infty} \operatorname{dim} R\left(E_{T_{n}}(\lambda-\delta)-E_{T_{n}}(\mu+\delta)\right) \\
& =\liminf _{n \rightarrow \infty} \operatorname{dim} R\left(E_{B_{n}}(\lambda-\delta)-E_{B_{n}}(\mu+\delta)\right)
\end{aligned}
$$

(see [6, theorems 9.16.(i) and 9.19]). We have

$\operatorname{dim} R\left(E_{B_{n}}(\lambda-\delta)-E_{B_{n}}(\mu+\delta)\right)$

$$
\begin{aligned}
& \left.\left.=\operatorname{card}(\{t \in] \mu+\delta, \lambda-\delta] \mid \theta\left(t, \alpha_{n}, c_{n}, d_{n}\right)=\beta_{n} \bmod \pi\right\}\right), \\
& =\left[\frac{\theta\left(\lambda-\delta, \alpha_{n}, c_{n}, d_{n}\right)-\theta\left(\mu+\delta, \alpha_{n}, c_{n}, d_{n}\right)}{\pi}\right] \\
& \leqq\left[\frac{\theta\left(\lambda, \alpha_{n}, c_{n}, d_{n}\right)-\theta\left(\mu, \alpha_{n}, c_{n}, d_{n}\right)}{\pi}\right],
\end{aligned}
$$

because $\theta\left(t, \alpha_{n}, c_{n}, d_{n}\right)$ is increasing in $t[7$, theorem 16.1]. Therefore,

$$
\begin{aligned}
\operatorname{dim} R\left(E_{T}(\lambda-)-E_{T}(\mu)\right) & \leqq \liminf _{\delta \rightarrow 0} \operatorname{dim} R\left(E_{T}(\lambda-\delta)-E_{T}(\mu+\delta)\right) \\
& \leqq \liminf _{n \rightarrow \infty}\left[\frac{\theta\left(\lambda, \alpha_{n}, c_{n}, d_{n}\right)-\theta\left(\mu, \alpha_{n}, c_{n}, d_{n}\right)}{\pi}\right] .
\end{aligned}
$$

Proposition 1.2. If $T$ is any self-adjoint operator generated by $\tau$ on $\mathbb{R}$ and $-\infty<\mu<\lambda<+\infty$, then

$$
\operatorname{dim} R\left(E_{T}(\lambda)-E_{T}(\mu-)\right) \geqq \sup _{c<d, \alpha \in \mathbb{R}}\left[\frac{\theta(\lambda, \alpha, c, d)-\theta(\mu, \alpha, c, d)}{\pi}\right]-1 .
$$

Proof. Let us choose $c, d, \alpha$ in $\mathbb{R}$, and let us suppose that $c<d$ and

$$
n:=\left[\frac{\theta(\lambda, \alpha, c, d)-\theta(\mu, \alpha, c, d)}{\pi}\right] \geqq 2 .
$$

We introduce the operator

$$
B:\left\{\begin{array}{l}
D(B) \subset L^{2}(] c, d[, R) \rightarrow L^{2}(] c, d[, R), \\
B u=\tau u
\end{array}\right.
$$

with

$$
\begin{aligned}
\mid D(B)= & \left\{u \in L^{2}(] c, d[, R) \mid u \text { is loc. abs. cont., } \tau u \in L^{2}(] c, d[, R)\right. \text { and } \\
& \left.\sin (\alpha) u_{1}(c)-\cos (\alpha) u_{2}(c)=\sin (\beta) u_{1}(d)-\cos (\beta) u_{2}(d)=0\right\},
\end{aligned}
$$

where $\beta=\theta(\mu, \alpha, c, d)$. Since $\operatorname{dim} R\left(E_{B}(\lambda)-E_{B}(\mu-)\right)=\operatorname{card}(\{t \in[\mu, \lambda] \mid$ $\theta(t, \alpha, c, d)=\beta \bmod \pi\})=n+1$, there exists a subspace $M \subset R\left(E_{B}(\lambda)-E_{B}(\mu-)\right)$ such that (i) $\operatorname{dim}(M) \geqq n-1$, (ii) for all values of $u$ in $M: u(c)=u(d)=(0,0)$ and $\|\{B-(\lambda+\mu) / 2\} u\| \leq\{(\lambda-\mu) / 2\}\|u\|$. We can consider $M$ as a subspace of $D(T)$ and so we have $\operatorname{dim} R\left(E_{T}(\lambda)-E_{T}(\mu-)\right) \geqq n-1$ (if we had $\operatorname{dim} R\left(E_{T}(\lambda)-\right.$ $\left.E_{T}(\mu-)\right)<n-1$, then there would exist $f \in M$ such that $f \neq 0, f \perp R\left(E_{T}(\lambda)-\right.$ $\left.E_{T}(\mu-)\right)$ and $\left.\|\{T-(\lambda+\mu) / 2\} f\|>\{(\lambda-\mu) / 2\}\|f\|\right)$. 
Proposition 1.3. Let $u$ and $\tilde{u}$ be two non-trivial real solutions of $\tau u=\lambda u$ ( $\lambda$ in $\mathbb{R})$ and let $\theta$ and $\tilde{\theta}$ be any two determinations of the corresponding angular parts. If there exists $x_{0}$ in $\mathbb{R}$ and $k$ in $\mathbb{Z}$ such that $\tilde{\theta}\left(x_{0}\right)-\theta\left(x_{0}\right) \in[k \pi,(k+1) \pi[$, then for all values of $x \in \mathbb{R}: \tilde{\theta}(x)-\theta(x) \in[k \pi,(k+1) \pi[$.

Proof. We have

$$
\begin{aligned}
\sigma^{\prime}(x)= & \tilde{\theta}^{\prime}(x)-\theta^{\prime}(x)=g_{11}(x) \cos ^{2} \tilde{\theta}(x)+g_{22}(x) \sin ^{2} \tilde{\theta}(x) \\
& +2 g_{12}(x) \sin \tilde{\theta}(x) \cos \tilde{\theta}(x) \\
& -g_{11}(x) \cos ^{2} \theta(x)-g_{22}(x) \sin ^{2} \theta(x)-2 g_{12}(x) \sin \theta(x) \cos \theta(x) \\
= & \left\{g_{22}(x)-g_{11}(x)\right\}\left\{\sin ^{2} \tilde{\theta}(x)-\sin ^{2} \theta(x)\right\}+g_{12}(x)\{\sin 2 \tilde{\theta}(x)-\sin 2 \theta(x)\} \\
= & \left\{g_{22}(x)-g_{11}(x)\right\} \sin \{\tilde{\theta}(x)+\theta(x)\} \sin \sigma(x) \\
& +2 g_{12}(x) \cos \{\tilde{\theta}(x)+\theta(x)\} \sin \sigma(x),
\end{aligned}
$$

where $\sigma(x)=\tilde{\theta}(x)-\theta(x)$. This differential equation verifies the local existence and uniqueness theorem. Since $\sigma \equiv k \pi$ and $\sigma \equiv(k+1) \pi$ are solutions and $\sigma\left(x_{0}\right) \in[k \pi,(k+1) \pi[$, we have $\sigma(x) \in[k \pi,(k+1) \pi[$ for all $x$ in $\mathbb{R}$.

\section{Dirac systems with periodic and real coefficients}

We suppose that $q \equiv \frac{1}{2}$ and that $P$ and $R$ have the period $a>0 ; \tau$ becomes

$$
\tau u(x)=R(x)^{-1}\left\{\left(\begin{array}{rr}
0 & 1 \\
-1 & 0
\end{array}\right) u^{\prime}(x)+P(x) u(x)\right\} .
$$

For $\lambda$ in $\mathbb{R}$, let us introduce the fundamental system of solutions of $\tau u(x)=\lambda u(x)$ : $\varphi(x, \lambda), \psi(x, \lambda)$, satisfying

$$
\varphi(0, \lambda)=\left(\begin{array}{l}
1 \\
0
\end{array}\right) \text { and } \psi(0, \lambda)=\left(\begin{array}{l}
0 \\
1
\end{array}\right)
$$

We have

$$
W[\varphi, \psi](x, \lambda):=\left|\begin{array}{ll}
\varphi_{1}(x, \lambda) & \psi_{1}(x, \lambda) \\
\varphi_{2}(x, \lambda) & \psi_{2}(x, \lambda)
\end{array}\right| \equiv 1
$$

We introduce the discriminant, which is the real valued function defined by $D(\lambda)=\varphi_{1}(a, \lambda)+\psi_{2}(a, \lambda)(\lambda \in \mathbb{R})$.

For $\lambda \in \mathbb{R}$ such that $|D(\lambda)| \geqq 2$, let $\rho_{1}(\lambda)$ and $\rho_{2}(\lambda)$ in $\mathbb{R}$ be the two roots of $\rho^{2}-D(\lambda) \rho+1$ with $\left|\rho_{1}(\lambda)\right| \leqq 1 \leqq\left|\rho_{2}(\lambda)\right|$, and let $k(\lambda)$ in $\mathbb{C}$ satisfy $\exp \{a k(\lambda)\}=$ $\rho_{2}(\lambda)$. For $i=1,2$, there exists a real solution $e_{i}(x, \lambda)$ of $\tau u(x)=\lambda u(x)$ such that

$$
e_{i}(x+a, \lambda)=\rho_{i}(\lambda) e_{i}(x, \lambda),
$$

and if we define $z_{1}$ and $z_{2}$ by

$$
e_{1}(x, \lambda)=\exp \{-k(\lambda) x\} z_{1}(x, \lambda) \text { and } e_{2}(x, \lambda)=\exp \{k(\lambda) x\} z_{2}(x, \lambda),
$$

then $z_{i}(x+a, \lambda)=z_{i}(x, \lambda)$ for all $x$ in $\mathbb{R}$ and $i=1,2$.

If $\rho_{1}(\lambda) \neq \rho_{2}(\lambda)$, then $e_{1}(x, \lambda)$ and $e_{2}(x, \lambda)$ can be chosen linearly independent; the same is possible if $\rho_{1}(\lambda)=\rho_{2}(\lambda)(= \pm 1)$ and $\varphi_{2}(a, \lambda)=\psi_{1}(a, \lambda)=0$. If 
$\rho_{1}(\lambda)=\rho_{2}(\lambda)(= \pm 1)$ and $\left|\varphi_{2}(a, \lambda)\right|+\left|\psi_{1}(a, \lambda)\right|>0$, then we can choose $e_{i}(x, \lambda)$ such that $e_{1}(x, \lambda)=e_{2}(x, \lambda) \neq(0,0)$ (for all values of $x \in \mathbb{R}$ ), and there exists a solution $y(x)$ of $\tau u=\lambda u$, linearly independent of $e_{1}(x, \lambda)$, such that $\limsup _{x \rightarrow+\infty}|y(x)|=\limsup _{x \rightarrow-\infty}|y(x)|=+\infty$.

We can take for example

$$
\begin{aligned}
& e_{i}(x, \lambda)=\psi_{1}(a, \lambda) \varphi(x, \lambda)+\left\{\rho_{i}(\lambda)-\varphi_{1}(a, \lambda)\right\} \psi(x, \lambda), \text { or } \\
& e_{i}(x, \lambda)=\left\{\rho_{i}(\lambda)-\psi_{2}(a, \lambda)\right\} \varphi(x, \lambda)+\varphi_{2}(a, \lambda) \psi(x, \lambda),
\end{aligned}
$$

but these functions can be null $(i=1,2)$. Note that they are analytic in $\lambda$ on $\{\lambda \in \mathbb{R}|| D(\lambda) \mid>2\}$ for every $x \in \mathbb{R}$. The reader is referred to [2] and [7] for more information.

The maximal operator defined on $\mathbb{R}$ by $\tau$ is self-adjoint and its spectrum is equal to $\{\lambda \in \mathbb{R}|| D(\lambda) \mid \leqq 2\}$. Let $] \mu, v\left[\right.$ be a gap of its spectrum and let $e_{\mu}$ and $e_{v}$ be two non-trivial, real and periodic solutions of, respectively, $\tau u=\mu u$ and $\tau u=v u$. We shall denote any two determinations of the corresponding angular parts by $\theta_{\mu}$ and $\theta_{v}$. Let $c<d$ be in $\mathbb{R}$ such that $d-c$ is in a $\mathbb{Z}$.

Proposition 2.1. (1) $\theta_{v}(d)-\theta_{\mu}(d)=\theta_{v}(c)-\theta_{\mu}(c)$;

(2) if $\theta$ is the determination of the angular part of a non-trivial real solution of $\tau u=v u$ such that $\theta(c)=\theta_{\mu}(c)$, then $\left.\theta(d)-\theta_{\mu}(d) \in\right] 0, \pi[$.

Proof. (1) Since $\theta_{\mu}(x)$ and $\theta_{v}(x)$ are the angular parts of periodic or semi-periodic functions of period or semi-period $a$, there exists $k$ in $\mathbb{Z}$ such that $\left\{\theta_{v}(d)-\theta_{\mu}(d)\right\}-\left\{\theta_{v}(c)-\theta_{\mu}(c)\right\}=k \pi$.

Case (i). Let us suppose that $k>0$. For $n \in \mathbb{N}$, set $c_{n}=c-(d-c) n$ and $d_{n}=d+(d-c) n$. Then $\left\{\theta_{v}\left(d_{n}\right)-\theta_{\mu}\left(d_{n}\right)\right\}-\left\{\theta_{v}\left(c_{n}\right)-\theta_{\mu}\left(c_{n}\right)\right\}=(2 n+1) k \pi$. Let $\theta_{n}$ be the determination of the angular part of a non-trivial real solution of $\tau u=v u$ such that $\theta_{n}\left(c_{n}\right)=\theta_{\mu}\left(c_{n}\right)$. We have

$$
\begin{aligned}
\theta_{n}\left(d_{n}\right)-\theta_{\mu}\left(d_{n}\right)= & \left(\left\{\theta_{n}\left(d_{n}\right)-\theta_{v}\left(d_{n}\right)\right\}-\left\{\theta_{n}\left(c_{n}\right)-\theta_{v}\left(c_{n}\right)\right\}\right) \\
& +\left(\left\{\theta_{v}\left(d_{n}\right)-\theta_{\mu}\left(d_{n}\right)\right\}-\left\{\theta_{v}\left(c_{n}\right)-\theta_{\mu}\left(c_{n}\right)\right\}\right) .
\end{aligned}
$$

By Proposition 1.3, the first term belongs to ] $-\pi, \pi[$ and therefore

$$
\left.\theta_{n}\left(d_{n}\right)-\theta_{\mu}\left(d_{n}\right) \in\right](2 n+1) k \pi-\pi, \quad(2 n+1) k \pi+\pi[.
$$

By Proposition 1.2, there is an infinity of eigenvalues of $T$ in $[\mu, v]$. This assertion being false, we have proved that $k \leqq 0$.

Case (ii). Let us suppose that $k<0$. Then $\theta_{n}\left(d_{n}\right)-\theta_{\mu}\left(d_{n}\right)<0$ for $n \in \mathbb{N}$. Hence we have a contradiction with [7, theorem 16.1].

(2) There exists $k$ in $\mathbb{Z}$ such that $\theta(c)-\theta_{v}(c)=\theta_{\mu}(c)-\theta_{v}(c)=\theta_{\mu}(d)-$ $\theta_{v}(d) \in\left[k \pi,(k+1) \pi\left[\right.\right.$. By Proposition 1.3, $\theta(d)-\theta_{v}(d) \in[k \pi,(k+1) \pi[$. Thus

$$
\left.\theta(d)-\theta_{\mu}(d)=\left\{\theta(d)-\theta_{v}(d)\right\}-\left\{\theta_{\mu}(d)-\theta_{v}(d)\right\} \in\right]-\pi, \pi[.
$$

By [7, theorem 16.1], $\theta(d)>\theta_{\mu}(d)$ and therefore $\left.\theta(d)-\theta_{\mu}(d) \in\right] 0, \pi[$. 


\section{The main results}

Let $\tau$ and $\tilde{\tau}$ be as in the Introduction and let $] \mu, v$ [ be a gap of $\sigma_{e}(\tilde{T})$ (we suppose that there is at least one gap).

Proposition 3.1. If for $N \in \mathbb{N}$,

$$
\int_{A}^{B}\left\{(|\mu|+|v|)\left(r_{2}(x)-r_{1}(x)\right)+2\left(p_{2}(x)-p_{1}(x)\right)+\left(\delta p_{2}(x)-\delta p_{1}(x)\right)\right\} d x \leqq N \pi,
$$

then there are at most $(N+1)$ eigenvalues of $\tilde{T}$ in $] \mu, v[$.

Proof. Let $e_{\mu}$ be a non-trivial, real and periodic solution of $\tau u=\mu u$, and let $\theta_{\mu}$ be any determination of its angular part. For $n \in \mathbb{N}$, we introduce the following notation: $\theta$ is the determination of the angular part of a real non-trivial solution of $\tau u=v u$ such that $\theta(-n a)=\theta_{\mu}(-n a)$; $\tilde{\theta}_{\mu}$ is the determination of the angular part of a real non-trivial solution of $\tilde{\tau} u=\mu u$ such that $\tilde{\theta}_{\mu}(-n a)=\theta_{\mu}(-n a) ; \tilde{\theta}$ is the determination of the angular part of a real non-trivial solution of $\tilde{\tau} u=v u$ such that $\tilde{\theta}(-n a)=\theta_{\mu}(-n a)$.

We have

$$
\begin{aligned}
& \theta_{\mu}^{\prime}(x)=\left((\mu R(x)-P(x))\left(\begin{array}{c}
\cos \theta_{\mu}(x) \\
\sin \theta_{\mu}(x)
\end{array}\right),\left(\begin{array}{c}
\cos \theta_{\mu}(x) \\
\sin \theta_{\mu}(x)
\end{array}\right)\right), \\
& \tilde{\theta}_{\mu}^{\prime}(x)=\left((\mu R(x)-P(x)-\Delta P(x))\left(\begin{array}{l}
\cos \tilde{\theta}_{\mu}(x) \\
\sin \tilde{\theta}_{\mu}(x)
\end{array}\right),\left(\begin{array}{c}
\cos \tilde{\theta}_{\mu}(x) \\
\sin \tilde{\theta}_{\mu}(x)
\end{array}\right)\right), \\
& \theta^{\prime}(x)=\left((v R(x)-P(x))\left(\begin{array}{c}
\cos \theta(x) \\
\sin \theta(x)
\end{array}\right),\left(\begin{array}{c}
\cos \theta(x) \\
\sin \theta(x)
\end{array}\right)\right), \\
& \tilde{\theta}^{\prime}(x)=\left((v R(x)-P(x)-\Delta P(x))\left(\begin{array}{c}
\cos \tilde{\theta}(x) \\
\sin \tilde{\theta}(x)
\end{array}\right),\left(\begin{array}{c}
\cos \tilde{\theta}(x) \\
\sin \tilde{\theta}(x)
\end{array}\right)\right),
\end{aligned}
$$

and thus

$$
\begin{aligned}
\mid\left\{\tilde{\theta}^{\prime}(x)-\theta^{\prime}(x)\right\}- & \left\{\tilde{\theta}_{\mu}^{\prime}(x)-\theta_{\mu}^{\prime}(x)\right\} \mid \\
& \leqq(|\mu|+|v|)\left(r_{2}(x)-r_{1}(x)\right)+2\left(p_{2}(x)-p_{1}(x)\right)+\left(\delta p_{2}(x)-\delta p_{1}(x)\right) .
\end{aligned}
$$

Let us suppose that $n$ is such that $[A, B] \subset]-n a, n a[$ and let $k$ be in $\mathbb{Z}$ such that $\tilde{\theta}_{\mu}(B)-\theta_{\mu}(B) \in[k \pi,(k+1) \pi[$. We have

$$
\begin{aligned}
&\left|\{\tilde{\theta}(B)-\theta(B)\}-\left\{\tilde{\theta}_{\mu}(B)-\theta_{\mu}(B)\right\}\right| \\
& \leqq \int_{A}^{B}\left\{\tilde{\theta}^{\prime}(x)-\theta^{\prime}(x)\right\}-\left\{\tilde{\theta}_{\mu}^{\prime}(x)-\theta_{\mu}^{\prime}(x)\right\} \mid d x \leqq N \pi
\end{aligned}
$$

and therefore $\tilde{\theta}(B)-\theta(B) \in[(k-N) \pi, \quad(k+N+1) \pi[$. By Proposition 1.3, $\tilde{\theta}_{\mu}(n a)-\theta_{\mu}(n a) \in[k \pi,(k+1) \pi[$ and $\tilde{\theta}(n a)-\theta(n a) \in[(k-N) \pi,(k+N+1) \pi[$. Using $\left.\theta(n a)-\theta_{\mu}(n a) \in\right] 0, \pi[$ (Proposition 2.1 (2)), we get

$$
\begin{aligned}
\tilde{\theta}(n a)-\tilde{\theta}_{\mu}(n a)= & \{\tilde{\theta}(n a)-\theta(n a)\}-\left\{\tilde{\theta}_{\mu}(n a)-\theta_{\mu}(n a)\right\} \\
& +\left\{\theta(n a)-\theta_{\mu}(n a)\right\}<(k+N+1-k+1) \pi=(N+2) \pi .
\end{aligned}
$$


Letting $n$ tend to $+\infty$, the result now follows from Proposition 1.1 and the fact that the eigenvalues are of multiplicty one (for $\lambda \in] \mu, v\left[\right.$, a solution in $L^{2}(\mathbb{R}, R$ ) of $\tilde{\tau} u=\lambda u$ is a multiple of $e_{1}(., \lambda)$ on $\left[B, \infty\left[\right.\right.$ and a multiple of $e_{2}(., \lambda)$ on ] $-\infty, A])$.

Proposition 3.2. If

$$
\begin{gathered}
\int_{A}^{B}\left\{\max (|\mu|,|v|)\left(r_{2}(x)-r_{1}(x)\right)+\left(p_{2}(x)-p_{1}(x)\right)+\left|\delta p_{2}(x)\right|\right\} d x \leqq \pi / 2, \\
\delta p_{1}(x)=\delta p_{2}(x) \quad \text { almost everywhere on } \mathbb{R},
\end{gathered}
$$

$\delta p_{2}$ is not equal almost everywhere to the null function,

$\delta p_{2}(x) \geqq 0 \quad$ almost everywhere or $\delta p_{2}(x) \leqq 0 \quad$ almost everywhere on $\mathbb{R}$, then there is exactly one eigenvalue of $\tilde{T}$ in $] \mu, v[$.

Proof. We shall adapt a method of Rofe-Beketov [4]. Let $\varphi(x, \lambda)$ and $\psi(x, \lambda)$ be as in Section 2 and let $\tilde{\varphi}(x, \lambda)$ and $\tilde{\psi}(x, \lambda)$ be two solutions of $\tilde{\tau} u(x)=\lambda u(x)$ satisfying

$$
\tilde{\varphi}(0, \lambda)=\left(\begin{array}{l}
1 \\
0
\end{array}\right) \text { and } \tilde{\psi}(0, \lambda)=\left(\begin{array}{l}
0 \\
1
\end{array}\right) .
$$

Let us introduce the two regular matrices

$$
L(x, \lambda)=(\varphi(x, \lambda) \psi(x, \lambda)) \text { and } \tilde{L}(x, \lambda)=(\tilde{\varphi}(x, \lambda) \tilde{\psi}(x, \lambda)),
$$

let $v$ and $w$ be two real solutions of $\tau u=\lambda u(\lambda \in \mathbb{R}$ is fixed) and let $\tilde{v}$ and $\tilde{w}$ be two real solutions of $\tilde{\tau} u=\lambda u$, such that $v$ and $\tilde{v}$ are equal on $[B,+\infty[$, and $w$ and $\tilde{w}$ are equal on $]-\infty, A]$.

Using the method of variation of constants, we get

$$
\begin{aligned}
& \tilde{w}(x)=w(x)+L(x, \lambda) \int_{A}^{x}\left\{L^{-1}(t, \lambda)\left(\begin{array}{rr}
0 & 1 \\
-1 & 0
\end{array}\right) \Delta P(t) \tilde{w}(t)\right\} d t \\
& v(x)=\tilde{v}(x)-\tilde{L}(x, \lambda) \int_{B}^{x}\left\{\tilde{L}^{-1}(t, \lambda)\left(\begin{array}{rl}
0 & 1 \\
-1 & 0
\end{array}\right) \Delta P(t) v(t)\right\} d t,
\end{aligned}
$$

and using

$$
v^{t}(x)\left(\begin{array}{rr}
0 & 1 \\
-1 & 0
\end{array}\right) L(x, \lambda)=v^{t}(t)\left(\begin{array}{rr}
0 & 1 \\
-1 & 0
\end{array}\right) L(t, \lambda)
$$

and

$$
\tilde{w}^{t}(x)\left(\begin{array}{rr}
0 & 1 \\
-1 & 0
\end{array}\right) \tilde{L}(x, \lambda)=\tilde{w}^{t}(t)\left(\begin{array}{rr}
0 & 1 \\
-1 & 0
\end{array}\right) \tilde{L}(t, \lambda)
$$

we obtain

$$
\begin{aligned}
W[\tilde{v}, \tilde{w}] & =W[v, w]+v^{t}\left(\begin{array}{rr}
0 & 1 \\
-1 & 0
\end{array}\right)(\tilde{w}-w)-\tilde{w}^{t}\left(\begin{array}{rr}
0 & 1 \\
-1 & 0
\end{array}\right)(\tilde{v}-v) \\
& =W[v, w]-\int_{A}^{B}(\Delta P(t) v(t), \tilde{w}(t)) d t .
\end{aligned}
$$


Now let us consider the case $v=e_{1}(x, \lambda)$ and $w=e_{2}(x, \lambda)$, where $e_{1}(x, \lambda)$ and $e_{2}(x, \lambda)$ are defined by one of the formulae (2.1) and $\lambda \in[\mu, v]$. We shall use $\dot{u}$ for the derivation in $\lambda, u^{\prime}$ for the derivation in $x$, and $E_{i}(x, \lambda)$ for $\tilde{e}_{i}(x, \lambda)$. Since $d / d x\left(E_{12} \dot{E}_{21}-E_{11} \dot{E}_{22}\right)=d / d x\left(E_{22} \dot{E}_{11}-E_{21} \dot{E}_{12}\right)=-\left(R E_{1}, E_{2}\right)$, we have

$$
\begin{aligned}
\frac{d}{d \lambda} W\left[E_{1}, E_{2}\right](\Lambda) & =\left\{\left(E_{22} \dot{E}_{11}-E_{21} \dot{E}_{12}\right)-\left(E_{12} \dot{E}_{21}-E_{11} \dot{E}_{22}\right)\right\}(0, \Lambda) \\
& =\int_{-\infty}^{+\infty}\left(R(t) E_{1}(t, \Lambda), E_{2}(t, \Lambda)\right) d t \neq 0
\end{aligned}
$$

if for all values of $t \in \mathbb{R}: E_{1}(t, \Lambda)=E_{2}(t, \Lambda) \neq(0,0)$, i.e. if $\left.\Lambda \in\right] \mu, v[$ is an eigenvalue of $\tilde{T}$ such that $W\left(e_{1}, e_{2}\right)(\Lambda) \neq 0$.

Set $e_{i}(x, \lambda)=\psi_{1}(a, \lambda) \varphi(x, \lambda)+\left\{\rho_{i}(\lambda)-\varphi_{1}(a, \lambda)\right\} \psi(x, \lambda)$. We have

$$
W\left[e_{1}, e_{2}\right](\lambda)=\psi_{1}(a, \lambda)\left\{\rho_{2}(\lambda)-\rho_{1}(\lambda)\right\} \neq 0
$$

if $\lambda \in] \mu, v\left[\right.$ and $\psi_{1}(a, \lambda) \neq 0$.

As for Sturm-Liouville operators with Dirichlet and Neumann boundary conditions [7, chap. 13], the spectrum of the operator generated by $\tau$ on $] 0, a[$ with boundary conditions $u_{2}(0)=u_{2}(a)=0$ (respectively $u_{1}(0)=u_{1}(a)=0$ ) is equal to $\left\{\lambda \mid \varphi_{2}(a, \lambda)=0\right\}$ (respectively $\left\{\lambda \mid \psi_{1}(a, \lambda)=0\right\}$ ). We can also prove that in each maximal interval included in $\{\lambda|| D(\lambda) \mid \geqq 2\}, \varphi_{2}(a, \lambda)$ and $\psi_{1}(a, \lambda)$ have exactly one zero. In particular, there exists an unique $\kappa \in[\mu, v]$ such that $\psi_{1}(a, k)=0$.

Case (i). $\kappa$ is not an eigenvalue of $\tilde{T}$ and $\kappa \in] \mu, v[$. If $\lambda \in\{\mu, v\}$, we have

The hypothesis

$$
W\left[E_{1}, E_{2}\right](\lambda)=-\int_{A}^{B}\left(\Delta P(t) e_{2}(t, \lambda), E_{2}(t, \lambda)\right) d t .
$$

$$
\int_{A}^{B}\left\{\max (|\mu|,|v|)\left(r_{2}(x)-r_{1}(x)\right)+\left(p_{2}(x)-p_{1}(x)\right)+\left|\delta p_{2}(x)\right|\right\} d x \leqq \pi / 2
$$

implies that there are at most two eigenvalues in $] \mu, v[$ (Proposition 3.1) and, with the fact that $e_{2}$ and $E_{2}$ are not trivial, that the cosine of the angle between $e_{2}$ and $E_{2}$ is not negative on $[A, B]$. Indeed,

and

$$
\theta^{\prime}(x)=\left((\lambda R(x)-P(x))\left(\begin{array}{c}
\cos \theta(x) \\
\sin \theta(x)
\end{array}\right),\left(\begin{array}{c}
\cos \theta(x) \\
\sin \theta(x)
\end{array}\right)\right)
$$

$$
\tilde{\theta}^{\prime}(x)=\left((\lambda R(x)-P(x)-\Delta P(x))\left(\begin{array}{c}
\cos \tilde{\theta}(x) \\
\sin \tilde{\theta}(x)
\end{array}\right),\left(\begin{array}{c}
\cos \tilde{\theta}(x) \\
\sin \tilde{\theta}(x)
\end{array}\right)\right)
$$

thus

$$
\left|\tilde{\theta}^{\prime}(x)-\theta^{\prime}(x)\right| \leqq \max (|\mu|,|v|)\left(r_{2}(x)-r_{1}(x)\right)+\left(p_{2}(x)-p_{1}(x)\right)+\left|\delta p_{2}(x)\right|
$$

and, for all $x$ in $[A, B]$,

$$
\begin{aligned}
\mid \tilde{\theta}(x) & -\theta(x) \mid \\
& \leqq \int_{A}^{x}\left\{\max (|\mu|,|v|)\left(r_{2}(s)-r_{1}(s)\right)+\left(p_{2}(s)-p_{1}(s)\right)+\left|\delta p_{2}(s)\right|\right\} d s \leqq \pi / 2,
\end{aligned}
$$


where $\theta$ and $\tilde{\theta}$ are any two determinations of the angular parts of $e_{2}$ and $E_{2}$ respectively, such that $\theta(A)=\tilde{\theta}(A)$, and $\lambda \in\{\mu, v\}$. Since $\delta p_{1}=\delta p_{2}$ has a constant sign and is not equal almost everywhere to the null function,

$$
W\left[E_{1}, E_{2}\right](\mu)=-\int_{A}^{B}\left(\Delta P(t) e_{2}(t, \mu), E_{2}(t, \mu)\right) d t
$$

and

$$
W\left[E_{1}, E_{2}\right](v)=-\int_{A}^{B}\left(\Delta P(t) e_{2}(t, v), E_{2}(t, v)\right) d t
$$

are not null and have the opposite sign of $\delta p_{2}$. Moreover, the function $W\left[E_{1}, E_{2}\right](\lambda)$ crosses the $\lambda$-axis at $\lambda=\kappa$ and at every eigenvalue. Therefore, there is exactly one eigenvalue in $] \mu, v[$.

In order to prove that $W\left[E_{1}, E_{2}\right](\lambda)$ crosses the $\lambda$-axis at $\lambda=\kappa$, we introduce

and

$$
f_{b}(x, \lambda)=\psi_{1}(a, \lambda) \varphi(x, \lambda)+\left\{\rho_{b}(\lambda)-\varphi_{1}(a, \lambda)\right\} \psi(x, \lambda)
$$

$$
f_{c}(x, \lambda)=\left\{\rho_{c}(\lambda)-\psi_{2}(a, \lambda)\right\} \varphi(x, \lambda)+\varphi_{2}(a, \lambda) \psi(x, \lambda) .
$$

We suppose that $\{b, c\}=\{1,2\}$ and $\rho_{c}(\kappa) \neq \psi_{2}(a, \kappa)$. Let $F_{i}(x, \lambda)(i=1,2)$ be the corresponding perturbed functions such that $F_{1}(., \lambda)$ and $f_{1}(., \lambda)$ are equal on $\left[B, \infty\left[\right.\right.$, and $F_{2}(., \lambda)$ and $f_{2}(., \lambda)$ are equal on $\left.]-\infty, A\right]$. It follows that

$$
\begin{gathered}
W\left[f_{b}, f_{c}\right](\lambda)=\left\{\rho_{c}(\lambda)-\psi_{2}(a, \lambda)\right\}\left\{\rho_{c}(\lambda)-\rho_{b}(\lambda)\right\}, \\
W\left[e_{1}, e_{2}\right](\lambda)=\frac{\psi_{1}(a, \lambda)}{\rho_{c}(\lambda)-\psi_{2}(a, \lambda)} W\left[f_{1}, f_{2}\right](\lambda),
\end{gathered}
$$

and

$$
W\left[E_{1}, E_{2}\right](\lambda)=\frac{\psi_{1}(a, \lambda)}{\rho_{c}(\lambda)-\psi_{2}(a, \lambda)} W\left[F_{1}, F_{2}\right](\lambda)
$$

Near $\kappa, W\left[f_{b}, f_{c}\right](\lambda)$ and $W\left[F_{1}, F_{2}\right](\lambda)$ are not null, and $(\partial / \partial \lambda) \psi_{1}(a, \kappa) \neq 0$ (see below); therefore the function $W\left[E_{1}, E_{2}\right](\lambda)$ crosses the $\lambda$-axis at $\lambda=\kappa$.

Case (ii). $\kappa$ is an eigenvalue. Then $W\left[F_{1}, F_{2}\right](\lambda)$ and $\psi_{1}(a, \lambda)$ cross the $\lambda$-axis at $\lambda=\kappa$ and thus $W\left[E_{1}, E_{2}\right](\lambda)$ is zero at $\lambda=\kappa$ without crossing the $\lambda$-axis. The result follows in the same way as in case (i).

Case (iii). $\kappa \in\{\mu, v\}$. Let us introduce

$$
g_{i}(x, \lambda)=\left\{\rho_{i}(\lambda)-\psi_{2}(a, \lambda)\right\} \varphi(x, \lambda)+\varphi_{2}(a, \lambda) \psi(x, \lambda) \quad(i=1,2),
$$

and let $G_{i}(x, \lambda)$ be the corresponding perturbed functions such that $G_{1}(., \lambda)$ and $g_{1}(., \lambda)$ are equal on $\left[B, \infty\left[\right.\right.$, and $G_{2}(., \lambda)$ and $g_{2}(., \lambda)$ are equal on $\left.]-\infty, A\right]$. Since

$$
W\left[g_{1}, g_{2}\right](\lambda)=\varphi_{2}(a, \lambda)\left\{\rho_{1}(\lambda)-\rho_{2}(\lambda)\right\} \text {, }
$$

it follows that

$$
W\left[E_{1}, E_{2}\right](\lambda)=-\frac{\psi_{1}(a, \lambda)}{\varphi_{2}(a, \lambda)} W\left[G_{1}, G_{2}\right](\lambda)
$$


Moreover $\varphi_{2}(a, \kappa) \neq 0$ and $g_{1}(., \kappa)=g_{2}(., \kappa)$ is not trivial. Hence $W\left[G_{1}, G_{2}\right](\kappa) \neq 0$ and $W\left[G_{1}, G_{2}\right](\kappa)$ has the opposite sign of $\delta p_{2}$. Since

$$
\begin{aligned}
\frac{\partial}{\partial \lambda} \psi_{1}(a, \lambda)= & \int_{0}^{a}\left\{-(R(t) \psi(t, \lambda), \psi(t, \lambda)) \varphi_{1}(a, \lambda)\right. \\
& \left.+(R(t) \psi(t, \lambda), \varphi(t, \lambda)) \psi_{1}(a, \lambda)\right\} d t
\end{aligned}
$$

and

$$
\begin{aligned}
\frac{\partial}{\partial \lambda} \varphi_{2}(a, \lambda)= & \int_{0}^{a}\left\{(R(t) \varphi(t, \lambda), \varphi(t, \lambda)) \psi_{2}(a, \lambda)\right. \\
& \left.-(R(t) \psi(t, \lambda), \varphi(t, \lambda)) \varphi_{2}(a, \lambda)\right\} d t
\end{aligned}
$$

(see [2, lemma 2.1]), we have $(\partial / \partial \lambda) \varphi_{2}(a, \delta) \neq 0$, where $\delta$ is the unique zero of $\varphi_{2}(a, \lambda)$ in $[\mu, v]$, and $\operatorname{sgn}\left\{(\partial / \partial \lambda) \psi_{1}(a, \kappa)\right\}=-\operatorname{sgn}\left\{\varphi_{1}(a, \kappa)\right\}=-\operatorname{sgn}\{D(\kappa)\}=$ $-\operatorname{sgn}\{D(\delta)\}=-\operatorname{sgn}\left\{\psi_{2}(a, \delta)\right\}=-\operatorname{sgn}\left\{(\partial / \partial \lambda) \varphi_{2}(a, \delta)\right\}$, and thus $-\left(\psi_{1}(a, \lambda) /\right.$ $\left.\varphi_{2}(a, \lambda)\right)$ is negative between $k$ and $\lambda$. The results follows as in case (i).

Remarks 3.3. If $r_{1}=r_{2}$ and $\delta p_{1}=\delta p_{2}$, then Proposition 3.2 provides sufficient conditions for the perturbed operator to have exactly one eigenvalue in each gap, and Proposition 3.1 provides a sufficient condition on $\operatorname{supp}(\Delta P)$ for the perturbed operator to have at most $N+1$ eigenvalues in each gap $(N \in \mathbb{N})$.

\section{Acknowledgment}

I would like to thank S. D. Chatterji and C. A. Stuart for discussion and encouragement.

\section{References}

1 I. M. Glazman. Direct methods of qualitative spectral analysis of singular differential operators (Jerusalem: Israel program for scientific translations, 1965).

2 B. J. Harris. On the spectra and stability of periodic differential equations. Proc. London. Math. Soc. (3) 41 (1980), 161-192.

3 F. S. Rofe-Beketov. A test for the finiteness of number of discrete levels introduced into the gaps of a continuous spectrum by perturbations of a periodic potential. Soviet. Math. 5 (1964), 689-692.

4 F. S. Rofe-Beketov. Deficiency indices and properties of the spectrum of some classes of differential operators. In Spectral theory and differential equations, Lecture Notes in Mathematics 448, pp. 273-293 (Berlin: Springer, 1974).

5 E. Trubowitz. The inverse problem for periodic potentials. Comm. Pure. Appl. Math 30 (1977) 321-337.

6 J. Weidmann. Linear Operators in Hilbert Spaces, Graduate Texts in Mathematics 68 (Berlin: Springer, 1980).

7 J. Weidmann. Spectral theory of ordinary differential operators, Lecture Notes in Mathematics 1258 (Berlin: Springer 1987).

(Issued 21 August 1990) 